\title{
Lingüística contrastiva y lenguas cognadas en el contexto del aula de ELE en Brasil
}

\author{
JosÉ RIENDA \\ Universidad de Granada \\ NuRIA MaRÍA NiETo NúÑEZ \\ Universidade Federal do Rio Grande do Norte
}

Recepción: 20 Diciembre 2016 / Aceptación: 12 Septiembre 2017

ISSN: $1697-7467$

\begin{abstract}
RESUMEN: Está en nuestro propósito crear las condiciones idóneas que permitan a los futuros profesores de ELE alcanzar niveles de producción oral acordes con las necesidades propias de un profesor de lengua extranjera (LE) durante sus clases - de prácticas-. Tales clases han de servir no solo como herramienta de comunicación entre profesor-alumno, sino, especialmente, como modelo lingüístico significativo para sus propios alumnos. Así, la lingüística contrastiva y las lenguas cognadas serán claves en nuestro estudio. Además, conscientes de las peculiaridades que entrañan el aprendizaje de ELE por parte de aprendientes brasileños, un "termómetro" para medir su progresión son las interferencias.

Palabras clave: ELE, lenguas cognadas, lingüística contrastiva, interferencias lingüísticas.

\section{Contrastive Linguistics and Cognate Languages in the Context of SSL Class in Brazil}

ABSTRACT: Our purpose is to create the most profitable conditions that would allow the future teachers of SSL to reach oral production levels according to the specific needs that a foreign language teacher has during their classes - of practices-. This oral competence should allow them not only to use the Spanish language as a tool for communication between teacher-students, but, especially, as a significant linguistic model. In this way, the cognate languages and contrastive linguistics are very important. Aware of the peculiarities that involve learning SSL by Brazilian students, our "thermometer" for measuring that improvement will be the interferences.
\end{abstract}

Keywords: SSL, cognate languages, contrastive linguistics, linguistic interferences.

\section{Introducción: Las interferencias EN EL APRENDizaJe de ELE POR PARTE DE ESTUDIANTES BRASILEÑOS}

Las interferencias cobran una destacada importancia en el aprendizaje de LE desde el surgimiento de la Lingüística Contrastiva, la cual se encarga de predecir y analizar la comisión de errores en LE derivados de la influencia de la lengua materna (LM) en la fase de interlengua a través de la comparación entre lenguas. Este asunto se torna aún más controvertido cuando se trata de lenguas cognadas o afines, contexto en el que se sitúa nuestro trabajo -el aprendizaje de español por parte de futuros profesores de ELE nativos de portugués de Brasil-. Por lo tanto, se hace necesario reflexionar sobre dos puntos, que, a 
nuestro entender, son claves: la Lingüística Contrastiva -con énfasis en las interferencias- y la cuestión de las lenguas cognadas en el aprendizaje de LE por parte de aprendientes cuya LM es lingüísticamente próxima a la lengua objeto de estudio (LO).

\section{LA LiNGÜística CONTRASTIVA}

Para situar el nacimiento de la Lingüística Contrastiva (LC), tenemos que remontarnos a la II Guerra Mundial, cuando surgió el interés por buscar métodos más rápidos y eficaces para la enseñanza de lenguas extrajeras a los soldados estadunidenses, además de la atención a la expresión oral. A partir de ese interés, teorías, métodos y conocimientos lingüísticos comenzaron a trabajar en función de determinadas áreas de conocimiento en las que la lengua estaba involucrada, creando así nuevas disciplinas (enseñanza/aprendizaje de LE, logopedia, traducción asistida, lexicografía...). La Lingüística Aplicada, esto es, la aplicación de la Lingüística a otras áreas, ha destacado especialmente en el área de la enseñanza/aprendizaje de LE. Con este mismo objetivo, el de profundizar y mejorar el proceso de aprendizaje de LE, nace a finales de los años sesenta una subdisciplina de la Lingüística Aplicada: la Lingüística Contrastiva o lingüística de contrastes. Como afirma Vez Jeremías, la lingüística del contraste fue

un hito sustentado por el peso indudable que representan casi treinta años continuados de investigación aplicada en el entorno de la consecución de una teoría plausible, capaz de dar cuenta de lo que en realidad ocurre en ese encuentro de dos (o varios) sistemas lingüísticos diferentes en el interior de una única matriz cognitiva: la que posee el individuo que trata de apropiarse de más de una lengua (Vez, 2008: 151).

A lo largo de los años, se ha constatado cómo la LC ha representado un antes y un después en la enseñanza/aprendizaje de LE. La LC se fundamenta en la identificación de las diferencias y similitudes existentes, de forma sincrónica, entre dos, o más lenguas, sean estos elementos lingüísticos o socioculturales. La gran aportación radica en que estas diferencias son susceptibles de convertirse en una fuente de errores por parte de los aprendiente de la LO. Este hecho nos incita a la reflexión respecto a las dificultades y necesidades de los aprendientes brasileños del español y abre una serie de caminos que pueden ayudar a comprender aún mejor el proceso de aprendizaje para optimizar el rendimiento de los mismos.

En este sentido, las investigaciones concernientes a LC identifican los aspectos de la LO a los que debemos dar más énfasis o prestar especial atención e, indirectamente, aquellos que, por su absoluta equivalencia, representarían en teoría un facilitador para el aprendizaje. Consecuentemente, desde el punto de vista didáctico, nos permiten orientar más adecuadamente las metodologías, técnicas y estrategias de aprendizaje de LE aplicables, así como adaptar las programaciones de una forma más coherente. En este sentido Fente Gómez advierte de que

el papel de la lingüística de contrastes es, concretamente, el intentar racionalizar la enseñanza de lenguas extranjeras y, en especial, la introducción de mejoras sensibles en 
la programación de los cursos de escuelas e institutos de grado elemental y medio. La lingüística de contrastes se constituye, pues, en una parte importante de la lingüística aplicada con una clara finalidad didáctica y metodológica (Fente, 1980: 12).

Para tal efecto, los que se han destacado por reflejar estos aspectos contrastivos en sus contenidos son los materiales didácticos. Estos han recogido en mayor o menor medida dichos aspectos desde el inicio de la enseñanza de ELE en Brasil, ya en 1919, con la Gramática da língua espanhola para uso dos brasileiros de Nascentes (1920) y el Manual de Español de Becker (1945); hasta llegar a la actualidad (Español: curso de español para hablantes de Portugués, de Durão, en 2001; Expansión: español en Brasil, de Romanos y Carvahlo, en 2002; Espanhol: série Brasil, de Martin, en 2003; Español hoy, de López Barrera, en 2003; Español ahora, de Briones, en 2003; Espanhol para o ensino médio, de Palacios y Catino, 2004; Gente, de Martins y Sans Baulenas, en 2004; Español en marcha, de Castro, en 2005; Curso de español para profesionales brasileños, de Garrido, en 2007; Enlaces. Español para jóvenes brasileños, de Osman, en 2007; Agencia ELE, de Gil-Toresano, en 2009; Pasaporte ELE, de Cerrolaza, en 2010; Formación en español: lengua y cultura, de Villalba, en 2012).

Sin embargo, si observamos el Programa Nacional del Libro Didáctico del Ministerio de Educación y Cultura de Brasil, que se dedica a analizar anualmente los libros didácticos adoptados por las escuelas públicas brasileñas, se puede afirmar que los manuales de ELE para alumnos brasileños contemplan principalmente aspectos contrastivos léxicos (heterosemánticos) y en cierta medida sintácticos, pero, en menor medida, los aspectos fónicos (heterofónicos, heterotónico, entonación), morfológicos (ortografía, heteregenéricos), sin mencionar que los aspectos socioculturales quedan relegados a una mera exposición de contenidos informativos de países de habla hispana (Ferreira, 2006). Por todo ello, debemos destacar que la teoría y la práctica no caminan de la mano, porque las divergencias lingüísticas y, especialmente, las socioculturales no reciben la atención requerida.

Consideradas estas implicaciones, se hace necesario aclarar que, en términos generales, se ha de distinguir entre LC teórica y LC práctica, cuya diferencia fundamental está en el objetivo final de la investigación (Santos, 1993: 29). En este sentido, Fisiask (1995) sostiene que la primera estudia los conceptos y modelos que fundamentan la comparación para poder describir y comparar lenguas de forma pertinente, es decir, el ámbito de los universales lingüísticos; la segunda, por su parte, tiene un carácter más descriptivo, ya que basándose en los conceptos y modelos establecidos por la LC teórica, se encarga de realizar las comparaciones propiamente dichas entre dos o más lenguas y aplica sus hallazgos a la resolución de aspectos docentes, de traducción, etc.

A continuación, se analizan los tres modelos de análisis de la LC -el modelo de análisis contrastivo, el modelo de análisis de errores, el modelo de interlengua-, para así establecer lo que entendemos por interferencia. En este breve análisis, se puede observar cómo los cambios que introdujeron cada modelo representan una evolución desde el estructuralismo, pasando por el generativismo-transformacional, hasta la psicolingüística. Como afirma Santos Gargallo,

la observación empírica de la producción lingüística en hablantes no nativos (HNN), que se inicia a finales del decenio de los años sesenta del pasado siglo, viene motivada por el interés de los psicolingüistas en profundizar en el conocimiento del proceso 
de adquisición-aprendizaje de segundas lenguas (L2) y lenguas extranjeras (LE), una vez que fue puesta de manifiesto la insuficiencia del conductismo psicológico y del estructuralismo lingüístico y la parcialidad de los estudios contrastivos (Santos, 2008: 391).

\subsection{El modelo de análisis contrastivo: Interferencias}

Los principales investigadores del análisis contrastivo (AC), Fries (1945) y Lado (1957), sostenían que el aprendizaje de LE dependía de la capacidad de respuesta a estímulos que el aprendiente tiene y de los hábitos resultados de la misma. Dicho proceso de aprendizaje era el que habrían ensayado al aprender su LM. Desde esta visión conductista, el aprendiente transferiría sus 'conductas lingüísticas' adquiridas con su LM a la nueva lengua, lo que se convertiría en facilitador en los casos en que ambas lenguas compartiesen semejanzas lingüísticas, produciéndose así transferencias positivas. Söhrman (2007: 17) las clasifica en:

- enteramente formales (por ejemplo, dos ocurrencias del mismo sonido);

- homonímicas (la misma forma oral) u homógrafas (la misma forma visual, pero con significado diferentes);

- semánticas (el sentido coincide en su mayor parte);

- funcionales (tienen una misma función u objetivo).

A pesar del éxito que llegó a alcanzar el AC y el gran beneficio que didácticamente se puede obtener de él, se plantearon algunas críticas y limitaciones. Durão (2007) enumeró tales críticas y limitaciones en: uno) la idea de que la lengua no es un conjunto de hábitos automatizados; dos) la afirmación de que todas las estructuras diferentes de la LM invariablemente provocarían dificultades de aprendizaje; tres) la suposición de que la interferencia es el único factor que llevaba a los aprendices a cometer errores.

Cuando comenzó el declive del AC (Durão, 2007), Wardhaugh defendió la utilidad del modelo, aunque con un pequeño matiz: su aplicación debería de ser hecha a posteriori. Para este teórico, el problema del AC residía en que no se podían predecir los errores que cometerían los aprendientes sin compararlos con sus producciones, por eso propuso la versión débil -la que realizaba el AC era antes del proceso de aprendizaje- y la versión fuerte -que serviría para explicar los errores cometidos- (Wardhaugh, 1970), espacio en el que nos movemos para los análisis de discursos ya producidos por profesores de ELE en formación.

Las hipótesis del AC dieron lugar al concepto esencial de este modelo: las interferencias. Según Santos Gargallo, se dice que hay interferencias "cuando un individuo utiliza en una lengua meta (L2) un rasgo fonético, morfológico, sintáctico o léxico característico de su lengua nativa (L1)" (Santos, 1993: 35-36).

Por su parte, Weinrich y Haugen abrieron un nuevo escenario, que posteriormente se confirmaría con investigaciones de análisis de errores, afirmando que la LM no es la única razón generadora de interferencias, ya que la propia LE también puede ser la causante de errores (Weinrich 1953). Para distinguirlas, en el primer caso hablaremos de interferencias interlingüisticas y, en el segundo, de interferencias intralingüisticas.

Durão (2004: 81) clasifica las interferencias interlingüísticas en: 
a) la similitud fonológica u ortográfica;

b) la extensión por analogía;

c) la falta de habilidad para distinguir aspectos gramaticales de la LE con respecto a los de la LM;

d) el empleo de extranjerismos.

Las interferencias intralingüisticas, por su parte, comprenden aquellos errores derivados de la deducción por parte del aprendiente de las propias reglas de la LO, tales como la generalización, la analogía o la aplicación incorrecta de reglas. Esta clasificación de errores representan una gran dificultad para descubrir la real procedencia de los mismos, como afirma López Alonso y Seré:

La clasificación de errores intralinguales es interesante, pero no es fácil analizarlos; se trata de una propuesta de carácter descriptivo y no siempre es posible localizarlo y caracterizar ese error. El intento de llegar a esa conclusión, refleja, sin duda, un alto nivel de reflexión (López, 2002: 5).

Así mismo, Bustos Gisbert recoge esta dificultad y refleja la necesidad de establecer una taxonomía que los clasifique: "tal limitación tiene su origen en que carecemos de sistemas metodológicos con los que ordenar los errores de acuerdo con su origen y con la causa que los motiva; por ello, es necesario desarrollar modelos para la interpretación del significado de los errores" (Bustos, 1998: 18).

Esta necesidad es la que el Análisis de errores intentará suplir y pasamos a entender a continuación.

\subsection{El modelo de análisis de errores}

El modelo de análisis de errores (AE) irrumpe en la Lingüística Contrastiva con la trabajos de Corder en 1967, donde aparecen los primeros estudios sistemáticos de errores de los aprendientes de LE. Hasta el momento, los estudios que se venían realizando se limitaban a la comparación de los sistemas lingüísticos de dos lenguas, normalmente la LM y la LO, con el objetivo de encontrar las diferencias y similitudes existentes entre ambas para poder predecir las dificultades que encontrarían los aprendientes de la LO. Sin embargo, como demostraron numerosos investigadores, no todos los errores se debían a la influencia de la LM sobre el aprendizaje de la nueva lengua, por lo que, basándose en la teoría del cognitivismo chomskiana, se comienza a analizar los errores cometidos en producciones orales y escritas con el objetivo de entender cuáles son las estrategias cognitivas que están envueltas en el proceso de aprendizaje de una LE y se pasa a entender el error como algo positivo. En este sentido, los errores que aparecen en el uso de una lengua extranjera son elementos valiosos para el alumno, para el profesor y para el investigador por la información que proporcionan sobre el proceso de aprendizaje (Liceras, 1992:35).

Según esta nueva visión, el AE, las principales aportaciones según Corder (1967, 1973) se pueden formular de la siguiente manera: 
a) Podremos observar los paralelismos existentes entre el proceso de adquisición de la L1 y el proceso de aprendizaje de una L2, ya que ambos comparten algunos mecanismos, procedimientos y estrategias; y observaremos que el proceso de aprendizaje se ve facilitado por la experiencia previa de la adquisición.

b) La observación y el análisis de los errores en la producción lingüística dará cuenta de lo que el hablante no nativo interioriza y podremos establecer una clara diferencia entre la información lingüística a la que está expuesto (input) y la que procesa (intake).

c) La consideración de los errores es importante desde tres puntos de vista: primero, para el profesor, porque le orientará sobre lo que ha de enseñar; segundo, para el investigador, porque le aportará información sobre el proceso de aprendizaje y sobre los procesos psicológicos subyacentes al aprendizaje; y, tercero, para el que aprende, porque constatará sus hipótesis sobre el funcionamiento de la lengua objeto de aprendizaje (Santos, 2008: 395-396).

Consiguientemente, el error pasa a ser inevitable e importante dentro del proceso de adquisición de LE, tal y como él mismo definió en su artículo The significance of learners errors (1967). Los errores también van a caracterizar un sistema de lengua nuevo: el que tiene el aprendiente a lo largo de su proceso de aprendizaje y que recibe el nombre de dialecto idiosincrásico, sistema aproximativo o interlengua.

Corder propuso una dicotomía entre error sistemático -competencia incompleta- y error no sistemático -lapsos lingüísticos momentáneos-; el primero se derivaba de la competencia -conocimiento que se tiene de una lengua- y el segundo de la actuación, producto expuesto cuando el hablante emite una locución oral o escrita.

Entre la variada y compleja taxonomía para la clasificación de errores destacaremos los que siguen los criterios lingüísticos, gramatical, etiológico, pedagógico y comunicativo (Durão, 2007).

a) Criterio lingüístico. Según Burt y Kiparsky (1972) la clasificación por el criterio lingüístico se puede desarrollar de la siguiente manera:

- por adición: cuando se agrega un morfema o una palabra redundante o no adecuada en un determinado contexto;

- por omisión: cuando se suprime un morfema o alguna palabra imprescindible en un contexto;

- por ausencia de orden oracional (o por falsa colocación): refleja una organización inadecuada de constituyentes;

- por malformación de palabras o estructuras, por falsa elección / selección (cuando se usa un ítem de forma equivocada): indica una construcción inadecuada.

b) Criterio gramatical. Según Corder (1973), los errores deben ser analizados por niveles:

- error fonético-fonológico: afecta puntos referentes a confusiones causadas por oposiciones fonológicas que no existen en la lengua en cuestión o a confusiones motivadas por la existencia de sonidos diferentes en la lengua materna de los estudiantes; 
- error ortográfico: afecta a la forma gráfica de las palabras;

- error morfológico: atañe a la forma de las palabras;

- error sintáctico: atañe a la construcción de los enunciados;

- error léxico-semántico: afecta el significado de la lexías;

- error discursivo: afecta la constitución discursiva de los enunciados.

c) Criterio etiológico. Según Selinker (1972) y Richards (1973), toma como base la fuente de errores, subdividiéndolos en transitorios o de desarrollo -caracteriza los errores típicos de los estadios naturales de desarrollo- y permanentes -errores que tienden a mantenerse en la interlengua de los aprendientes-.

Estos errores pueden deberse a:

- transferencia (también denominados errores de interferencia o errores interlingüísticos): errores de producción, subproducción, superproducción, mala interpretación, hipercorrección.

- problemas intralingüísticos (también denominado error intralingual):

- por generalización (también llamados sobregeneralización o hipergeneralización): los aprendices extienden las reglas de la lengua meta a contextos inapropiados;

- por inducción: deriva de la forma en que un elemento lingüístico fue presentado a los aprendices y cómo lo practicaron;

- por producción excesiva (también designado subproducción): cuando los aprendices repiten determinadas estructuras, reiterándolas innecesariamente.

d) Criterio pedagógico. El criterio pedagógico refleja el tipo de producción de la que parte el que analiza, como se puede observar a continuación:

- de comprensión: cuando el objeto de análisis es una comprensión (oral / escrita);

- de producción: cuando el objeto de análisis es una producción (oral / escrita);

- individuales: cuando el objeto de análisis ha sido elaborado por un único aprendiz;

- colectivos: cuando el objeto de análisis ha sido elaborado por más de un aprendiz;

- orales: cuando el objeto de análisis es una producción oral;

- escritos: cuando el objeto de análisis es una producción escrita.

e) Criterio comunicativo. Los errores comunicativos se refieren a los que perturban diferentes situaciones comunicativas de alguna forma:

- global: afecta a todo el enunciado, de tal forma que no se puede comprender su sentido;

- local: afecta únicamente a algún constituyente o a algunas partes de un enunciado (Burt, 1972);

- irritante: causa una especie de 'enojo' porque afecta a estructuras de sentido común (Richards, 1973); 
- por inhibición: producido cuando se evitan ciertas estructuras de la lengua meta (Schachter, 1974);

- por ambigüedad: genera dificultades de comprensión debido a la existencia de ambigüedad (Vázquez, 1999);

- estigmatizador: tiene que ver con una impresión más o menos generalizada sobre una determinada comunidad de habla, induciendo a juicios negativos de valor (Richards, 1973);

- sociocultural: ocurre por transferencia de aspectos de un contexto lingüístico a otro, llevando a malos entendidos relacionados con significados socioculturalmente establecidos;

- pragmático: ocurre en función de la falta de entendimiento de un acto de habla o de alguna norma de habla, relacionado con la intención del hablante o con la falta de comprensión de tal intención por parte de su interlocutor.

Este modelo evolucionó y cambió sus objetivos a lo largo de su desarrollo. Así, de explicar y predecir tipos de errores de acuerdo con taxonomías gramaticales pasó a estudiar los efectos gramaticales y a valorar los errores en función de su efecto comunicativo, incorporando así el concepto de competencia comunicativa (Rienda, 2014: 761).

Por último, reconocemos que el AE representó un paso más en lo que a comprensión del aprendizaje de LE se refiere, aunque no está exento de críticas, especialmente en cuanto a la falta de precisión entre los términos 'descripción' y 'explicación' de las categorías y subcategorías de errores (Santos, 1993).

\subsection{El modelo de Interlengua}

Por necesidades evolutivas dentro de la LC y como se anunció en el AE, surgió el modelo de interlengua (IL) postulado por Selinker en 1972 (traducido en Liceras, 1992). A este respecto Santos Gargallo concluye que

el conjunto de oraciones que intenta reproducir un alumno que aprende una L2 no es idéntico al conjunto hipotetizado de las que produciría un hablante nativo de esa LO que intentara expresar los mismos significados que el alumno. Puesto que estos dos conjuntos de locuciones no son idénticos, cuando formulemos los principios relevantes para una teoría del aprendizaje de lenguas segundas estaremos completamente justificados, quizás hasta obligados, a presentar como hipótesis la existencia de un sistema lingüístico independiente: sobre la base de los datos observables que resultan de los intentos del alumno en la producción de una norma en la LO. Llamaremos 'interlengua' (IL) a este sistema lingüístico (Santos, 2008: 391).

A raíz de esta concepción de un sistema lingüístico en LO propio del alumno, este modelo intentará descubrir cómo procesa y usa el individuo aprendiente los datos lingüísticos de la LE que le llegan. Se distinguen tres procesos para la construcción del conocimiento interlingüístico: a) de aducto (input): seleccionar, ordenar y sistematizar los datos del exterior; b) de toma (intake): incorporar los nuevos datos del sistema; c) de educto (output): comprender y expresarse en la lengua meta (Baralo, 2008: 370). En consecuencia, el término 
interlengua "se refiere a los estadios que el aprendiz atraviesa antes de llegar al resultado final" (Fernández, 1997: 14).

Con esto, inferimos que "la interlengua es un sistema dinámico y continuo que cambia através de un proceso creativo" (Santo, 1993: 129) en el que el aprendiente hace uso de una serie de estrategias a lo largo de su proceso de IL, descritas por el lingüista Kim Griffin como sigue (Griffin, 2005: 92):

- Transferencia (de L1): realizar una "traducción literal".

- Sobregeneralización de reglas: puede dar lugar a "ultracorrección” por aplicar reglas indebidas en situaciones parecidas.

- Transferencia de instrucción: abuso generalizado de una regla recién aprendida.

- Estrategias de aprendizaje: diversas estrategias que el estudiante utiliza para asimilar el input y almacenar en su memoria.

- Estrategias de comunicación: se recurre a ellas cuando el aprendiente no es capaz de transmitir correctamente el mensaje con el objetivo de mantener la comunicación, para resolver problemas, etc.

Entre las aportaciones más destacadas que el modelo IL proporcionó a la LC se encuentra el concepto de fosilización, fenómeno lingüístico que caracteriza la interlengua y que hace mantener rasgos de la gramática de la LM en la estructura del sistema empleado por el aprendiente (Santos, 1993: 157).

Por último, hemos de concluir que cada modelo contrastivo no excluye al anterior, sino que se complementan. Cada uno difiere de los otros en sus principios metodológicos, datos analizados, resultados y propuestas de soluciones pedagógicas, pero todos ellos en busca de una enseñanza más eficiente de la LE. En términos generales, el modelo AC predice errores, el modelo AE cataloga y analiza los errores y el modelo IL indica, a través del tipo de error cometido por el aprendiente, en qué nivel de aprendizaje él se encuentra.

\section{Lenguas COGNAdas}

En la segunda mitad del siglo XVIII, L'Encyclopédie, bajo la dirección de Denis Diderot y Jean le Rond d'Alembert, es desarrollada con el objetivo de recoger y difundir todos los conocimientos y saberes acumulados hasta el momento. De ahí surge, entre otras cuestiones, la necesidad de clasificar las lenguas por familias, para lo cual se compararon por primera vez las lenguas con el propósito de establecer entre sí relaciones que pudieran justificar los orígenes de las mismas. Posteriormente, en el siglo XIX, florece la Lingüística Comparada -que no se debe confundir con la Lingüística Contrastiva- a partir de la cual podemos establecer el parentesco histórico entre determinadas lenguas.

Este es el caso del portugués y el español, que provienen del mismo tronco lingüístico, al ser ambas lenguas neolatinas o románicas. Por el hecho de compartir el mismo origen, el latín, poseen muchas equivalencias estructurales y coincidencias léxicas, pero, además, rasgos intransferibles y peculiaridades propias. Esto se debe al proceso evolutivo que experimentaron 
estas dos lenguas, donde inicialmente comparten una serie de rasgos como coincidencias fonéticas, fonológicas y sintácticas, para después, a partir de la segunda mitad del siglo XV y la primera del XVI, experimentar una serie de innovaciones que las separan y originan divergencias funcionales y semánticas, así como contrastes en el marco de la norma y el uso, esto es, divergencia progresiva (Ramírez, 2009). Estas divergencias y contrastes originados en España y Portugal peregrinaron hasta el cono sur de América.

Este contexto de enseñanza/aprendizaje de LE, el de lusohablantes aprendientes de español, acarrea unas consecuencias muy controvertidas, pues si por una parte presenta una serie de facilidades al aprendiente, pronto tales semejanzas desembocan en numerosos escollos. En este sentido, Martín Martín, a propósito de las aportaciones de Sweet y otros, sintetiza:

A finales del siglo pasado, Sweet escribía que si bien una lengua resulta más fácil cuanto más cercana es de nuestra L1, similitud con frecuencia es fuente de confusión y puede llegar a ser un obstáculo para llegar a un conocimiento profundo de la L2 si quien la aprende se conforma con entenderla y hablarla de manera suficiente, pero suficiente para una comunicación básica (Martín, 2000: 109).

Las facilidades se deben a la existencia de las similitudes mencionadas anteriormente, lo que dará lugar a 'falsos principiantes', pues aunque no hayan recibido instrucción alguna en dicha lengua, la semejanza interlingüística consigue crear una situación de partida muy peculiar, una especie de plataforma que sitúa al aprendiz en un nivel bastante más avanzado del que tendría en el caso de lenguas con mayor distancia. Durante este proceso de aproximación a la LO, el aprendiente interpreta esta proximidad como un pasaporte para transferir estructuras de la LM a la que está aprendiendo, evitando otras estrategias propias de cuando se está aprendiendo una LE, como la simplificación. Situados en la fase intermedia del aprendizaje, el aprendiente ya es consciente de las diferencias existentes y tiende a evitar dicha transferencia, pero llegado al estadio avanzado, la seguridad le lleva a hacer nuevamente uso de esa estrategia, lo que suele acarrear la fosilización de los errores.

En este sentido, el AC juega un papel fundamental, ya que tanto profesores como alumnos, ya sea consciente o inconscientemente, hacen uso de las transferencias e interferencias detectadas por este modelo contrastivo como técnica de aprendizaje. Siguiendo con el análisis interlingüístico, el AE aporta una clasificación de estos errores basados en una tentativa de entender las estrategias cognitivas que se dan en este proceso. Asimismo, Schmidt-Rinehat (1994) observa especialmente tres procedimientos: estrategia de congruencia que se basa en la observación de semejanzas entre la L1 y L2 y su consecuente trasvase de elementos de la primera a la segunda; estrategia de la correspondencia que establece reglas de conversión L1-L2 al observar regularidades en la L2, puesta en relación con la L1; estrategia de diferencia basada en el descubrimiento de divergencias interlingüísticas y el recurso a estructuras más distantes o inexistentes en la L1.

De acuerdo con nuestro contexto, destacamos las estrategias que señala Semino (2005) sobre las interferencias verbales: préstamos de sustitución, formas mezcladas, desinencias o raíces interferidas, calcos sintácticos, giros diferentes y presencia de tiempos no existentes o diferentes en español, así como algunos casos de hipergeneralización o sobregeneralización.

Desde un punto de vista gramatical, Silva Junior (2010) propone una clasificación en cuatro grupos: 1) concordancia entre los sujetos del discurso y las formas verbales: empleo 
de la conjugación verbal de la 2. ${ }^{a}$ persona en sustitución de la $3 .^{a}$ persona del singular; confusión entre la $3 .^{a}$ persona del singular y la $3 .^{a}$ del plural; 2) empleo del pronombre personal yo; 3) empleo de los pronombres personales: los sujetos coordinados; 4) verbos con irregularidades en la raíz: sobregeneralización del diptongo ie; omisión del diptongo ie; cambio de la vocal e por i; omisión del diptongo ue; sobregeneralización del diptongo ue.

A pesar de todas las consideraciones ya mencionadas, también está bastante extendida una idea más simplista basada en dos premisas en este contexto: es posible la comprensión, aunque se tenga poco o ningún conocimiento de la lengua y es posible establecer una comunicación 'exitosa'. Además, la realidad brasileña tiene como referencia del español una imagen que no contribuye a cambiar esta visión.

Con esta perspectiva, se crea el campo de cultivo para la propagación de lo que se conoce como 'portuñol' o 'portunhol', que se refiere a la mezcla de la lengua española y portuguesa, como ocurre con el 'spanglish'. Este término se suele usar para aquellos hablantes incompetentes, sean ellos lusohablantes o hispanohablantes, que nunca estudiaron la lengua o para denominar la fase de interlengua de un aprendiente. Se caracteriza, entre otras, por las siguientes incorrecciones (Bourguignon, 2007):

- Pobreza de vocabulario

- Desorden de ideas

- Uso de muletillas o sonorización intermedia de palabras

- Tono inadecuado

- Ideas poco claras, imprecisas

- Vocalización incorrecta.

Esta cuestión del portuñol está ampliamente tratada en las Orientaciones Curriculares para la Enseñanza Secundaria del Ministerio de Educación de Brasil (2006: 134-145) en el apartado 2, titulado "Algunas especificidades en la enseñanza de la Lengua española a estudiantes brasileños". En él se puede percibir que existe una conciencia de las particularidades que el aprendizaje plantea a los hablantes en Brasil y una preocupación por una enseñanza efectiva que acabe con el fenómeno del portuñol.

\section{Bibliografía}

Baralo Ottonello, M. (2008). "La interlengua del hablante no nativo", en J. S. Lobato e I. Santos Gargallo (eds.), Vademécum para la formación de profesores. Enseñar español como segunda lengua (L2) / lengua extranjera (LE). Madrid: SGEL, 369-390.

Becker, I. (1945). Manual de español. Sao Paulo: Editora Nacional.

Bourguignon, F., Fournier, M. and Gurgand, M. (2007). "Selection bias corrections based on the multinomial logit model: Monte Carlo comparisons", in Journal of Economic Surveys, 21: 174- 205.

Briones, A. I. et al. (2003). Español Ahora. Sao Paulo: Moderna.

Burt, M. K. and Kiparsky, C. (1972). The gooficon: a repair manual for English. Rowley: Newbury House Publishers.

Bustos Gisbert, J. M. (1998). La construcción de textos en español. Salamanca: Universidad de Salamanca. 
Castro Viúdez, F. et al (2005). Español en marcha. Madrid: SGEL.

Cerrolaza Aragón, M. et al (2010). Pasaporte ELE. Madrid: Edelsa.

Corder, S. P. (1967). "The Significance of Learners Errors", in IRAL, V, 4: 161-170.

Corder, S. P. (1973). Introducing Applied Linguistics. Harmondsworth: Penguin Books.

Durão, A. B. (2001). Español: curso de español para hablantes de portugués. Madrid: Arco Libros.

Durão, A. B. (2004). Análisis de errores e interlengua de lusohablantes aprendices de español y españoles aprendientes de portugués. Londrina: Editora da UEL.

Durão, A. B. (2007). La interlengua. Madrid: Arco Libros.

Fente Gómez, R. (1980). Lingüistica de contrastes. Granada: Universidad de Granada.

Fernández, S. (1997). Interlengua y Análisis de Errores en el aprendizaje del español como lengua extranjera. Madrid: Edelsa.

Ferreira, C. C. (2006). "La lingüística contrastiva y el proceso de enseñanza/aprendizaje de español como lengua extranjera", en Actas del II Simposio José Carlos Lisboa de Didáctica del Español para extranjeros. Rio de Janeiro: Instituto Cervantes: 191-201.

Fisiask, J. (1995). Contrastive linguistics and the language teacher. Elmsford: Pergamon Press.

Fries, Ch. (1945). Teaching and Learning English as a Foreign Language. Michigan: University of Michigan Press.

Garrido Esteban, G. et al (2007). Clave Curso de español para profesionales brasileños. Sao Paulo: Martins.

Gil-Toresano, M. et al (2009). Agencia ELE. Madrid: SGEL.

Griffin, K. (2005). Lingüistica aplicada a la enseñanza del español como segunda lengua. Madrid: Arco Libros.

Lado, R. (1957). Linguistics Across Cultures. Applied Linguistics for Language Teachers. Michigan: University of Michigan Press.

Liceras, J. M. (comp.) (1992). La adquisición de las lenguas extranjeras. Madrid: Visor.

López Alonso, C. Y Séré, A. (2002): "Hacia una nueva didáctica de la contrastividad en las LE: el enfoque congnitivo", en Carabela, 52: 5-21.

López Barberá, I. et al. (2003). Español Hoy. Sao Paulo: Scipione.

Martín Martín, J. M. (2000). La lengua materna en el aprendizaje de uma segunda lengua. Sevilla: Universidad de Sevilla.

Martin Peris, E. y Sans Baulenas, N. (2004). Gente. Barcelona: Difusión.

Martin, I. R. (2003). Espanhol: série Brasil. Sao Paulo: Ática.

Ministério da Ecucação (2006). Orientaciones Curriculares para la Enseñanza Secundaria. Río de Janeiro: Ministerio de Educación de Brasil.

Nascentes, A. (1920). Gramática de língua espanhola para o uso dos brasileiros. Rio de Janeiro: Pimenta de Mello.

Osman, S. et al. (2007). Enlaces. Español para jóvenes brasileños. Madrid: SGEL.

Palacios, M. y Catino, G. (2004). Espanhol para o Ensino Médio. Sao Paulo: Scipione.

Richards, J. C. (ed.) (1973). Error Analysis: perspectives in Second Languages Adquisition. London: Longman.

Rienda, J. (2014). "Límites conceptuales de la competencia literaria”, en Revista Signa, 23: 753-777.

Romanos, H. y Carvalho, J. P. (2002). Expansión: español en Brasil. Sao Paulo: FTD.

Santos Gargallo, I. (1993). Análisis contrastivo, análisis de errores e interlengua en el marco de la lingüistica contrastiva. Madrid: Síntesis.

Santos Gargallo, I. (2008). "El análisis de errores en la interlengua del hablante no nativo", en J. S. Lobato e I. Santos Gargallo (dir.), Vademécum para la formación de profesores. Enseñar español como segunda lengua (L2)/ lengua extranjera (LE). Madrid: SGEL: 391-410. 
Schachter, J. H. (1974). "An error in error analysis" in Language Learning, 24 (2): 205-14.

Schmidt-Rimehaut, B. (1994). "The effect of topic familiarity on second language listening comprehension", in The Modern Language Journal, 78: 179-221

Selinker, L. (1972). "Interlanguage", in IRAL, 10: 209-231.

Semino, M. a J. (2005). Español y Portugués: desenredando las lenguas. Guía para profesores y alumnos brasileños. Rio Grande: FURG.

Silva Júnior, P. A. (2010). Análisis de errores. Estudio de las estructuras verbales y discursivas en el aprendizaje del español/LE por parte de alumnos brasileños (producción escrita). Salamanca: Universidad de Salamanca.

Söhrman, I. (2007). La lingüistica contrastiva como herramienta para la enseñanza de lenguas. Madrid: Arco Libros.

Vázquez, G. (1999). ¿ERRORES? ¡Sin faltas! Madrid: Edelsa.

Vez Jeremías, J. M. (2004). "Aportaciones de la lingüística contrastiva", en J. S. Lobato e I. Santos Gargallo (dir.), Vademécum para la formación de profesores. Enseñar español como segunda lengua (L2)/ lengua extranjera (LE). Madrid: SGEL: 147-163.

Villalba, T. K. B. et al. (2012). Formación en español: lengua y cultura. Curitiba: Base Editorial. Wardaugh, U. (1970). "The Contrastive Analysis Hypothesis", in TESOL Quartely, 4: 123-130.

Weinreich, U. (1953). Languages in Contact. The Hauge: Mouton. 